\title{
Sonographic Diagnosis in Intestinal Ascariasis
}

\author{
M SAJJAD **M S QURESHI ***N R ZAIDI \\ Ibdullah Hospital Skardu. Baltistan. \\ - Maarafi Foundation Hospital Mehdiabad Skardu. Baltistan. \\ "Department of Diagnostic Radiology and Medical Imaging. Allama Iqbal Medical College I Jinnah Hospital. Lahore. \\ arrespondence to Dr. Malid. Sajjad
}

Ascaris Lumbricoides belongs to Nemathelminthes group and is one of the largest human nematode, which entrenches in the small intestine. Abdominopelvic sonographic evaluation of 350 patients was done at Medical Camp "Skardu" Baltistan, Pakistan, with features of "RAP" recurrent abdominal pain, dyspepsia, urinary retention, dysmenorrhea, biliary and renal colic etc. In 15 patients apart from other findings, linear, curved, tramline like echogenic structures were seen in the small intestine and stomach without any acoustic enhancement or shadowing. Cases with similar findings were also detected at Jinnah Hospital, Lahore. Findings were confirmed on barium study. According to WHO estimate 1.3 billion population is having worm infestation, Hence stressing upon the efficacy and importance of ultrasonography in diagnosis of intestinal ascariasis due to its easy availability, inexpensive and being non invasive.

Key Words: RAP: Recurrent abdominal pain, USG: ultrasonography, asçaris lumbricoides.

Ascaris is the most common and largest human intestinal nematode giving rise to variety of medical problems. Somewhat resembles the ordinary earthworm, but is unsegmented and brownish yellow in colour. Male worm little smaller than female and measures in length about $25 \mathrm{~cm}$ and breadth of $4-6 \mathrm{~mm}$. Posterior extremity is pointed and curved ventrally in the form of a hook. Female measures about $35 \mathrm{~cm}$ in length and width of about $4-6 \mathrm{~mm}$. Posterior extremity though conical neither pointed nor ventrally curved but straight. The eggs are resistant to cold and drying and to disinfectants. They develop in warmth $20-33^{\circ} \mathrm{C}$ and moisture and can remain alive and unhatched for months. They are killed if exposed to temperatures of about $45^{\circ} \mathrm{C}$.

Geographic distribution is cosmopolitan but more common in rural areas where hygiene is poor. Transmission typically occurs via faecally contaminated soil. by swallowing water or raw vegetables polluted with eggs and containing the infective larvae. Eggs pass through the stomach, larvae hatch in the intestine invade the mucosa migrate via the circulation are carried to right side of the heart, and finally to the lungs where they remain in the alveoli for several days during which they grow and moult twice. They are then carried up the trachea, pass over to the epiglottis and down to the oesophagus to the stomach and small intestine and develop into adult worm in about two months time. Worm can live for several vears. WHO estimate 1.3 billion persons or a quarter of human race are infected by this nematode. Every year up to a million cases of clinical disease are recorded and between 80.000 to 100.000 infested children die from intestinal obstruction and other abdominal complications of ascariasis.

In Skardu, Pakistan water supply system is from "SATPARA LAKE" where the catchments area is unprotected. In rural areas people divert water channels to their homes utilize and effluent is again mixing in other lakes. So the cycle of recontamination is continuing.

Materials and methods

While performing routine abdomino-pelvic sonographic examination with Sonoace, Capasee II and Tosbee machines with $3.75 \mathrm{MHz}$ convex \& $7.0 \mathrm{MHz}$ linear probes. Apart from other pathologies 15 cases presenting with complaints of pallor, recurrent abdominal pain. dyspepsia and loss of appetite were first provisionally considered normal. when major viscera like liver, spleen. Gall Bladder, pancreas, kidneys and urinary bladder were found unremarkable. Sonographic survey of bowel by magnifying the system revealed curled up or linear hyperechoic tramline like structure in small intestine and stomach at Skardu as well as in JHL, Pakistan. Characteristic movements were also exhibited in 5 cases by these echogenic tubular structures in bowel loops.

\section{Results}

Sonographic appearances in cases of this nematode were (a) relatively dilated bowel segment (b) linear tram line like structures (c) coiled up masses (d) echogenic tubular filling defects (e) disintegrated masses subsequent to oral treatment.

\section{Discussion}

Ascaris lumbricoides is the largest intestinal nematode parasite of the humans. During the pulmonary phase of larval cycle, patient can develop repeated bouts of cough non-productive and substernal discomfort. Where as in established case, when there is adult worm in the small intestine usually cause no symptoms. But in heav! infestation may cause colicky abdominal pain and features of obstruction mostly in children. Contrast studies can prove good tools to demonstrate worm and their complications in bowel loops provided there is no limitation. On barium study a tubular filling defect in the 
long axis of bowel or coiled structure is seen. Occasionally, the gastrointestinal tract of the worm is visualized on Barium examination when the parasite himself ingests the contrast. In situations when contrast
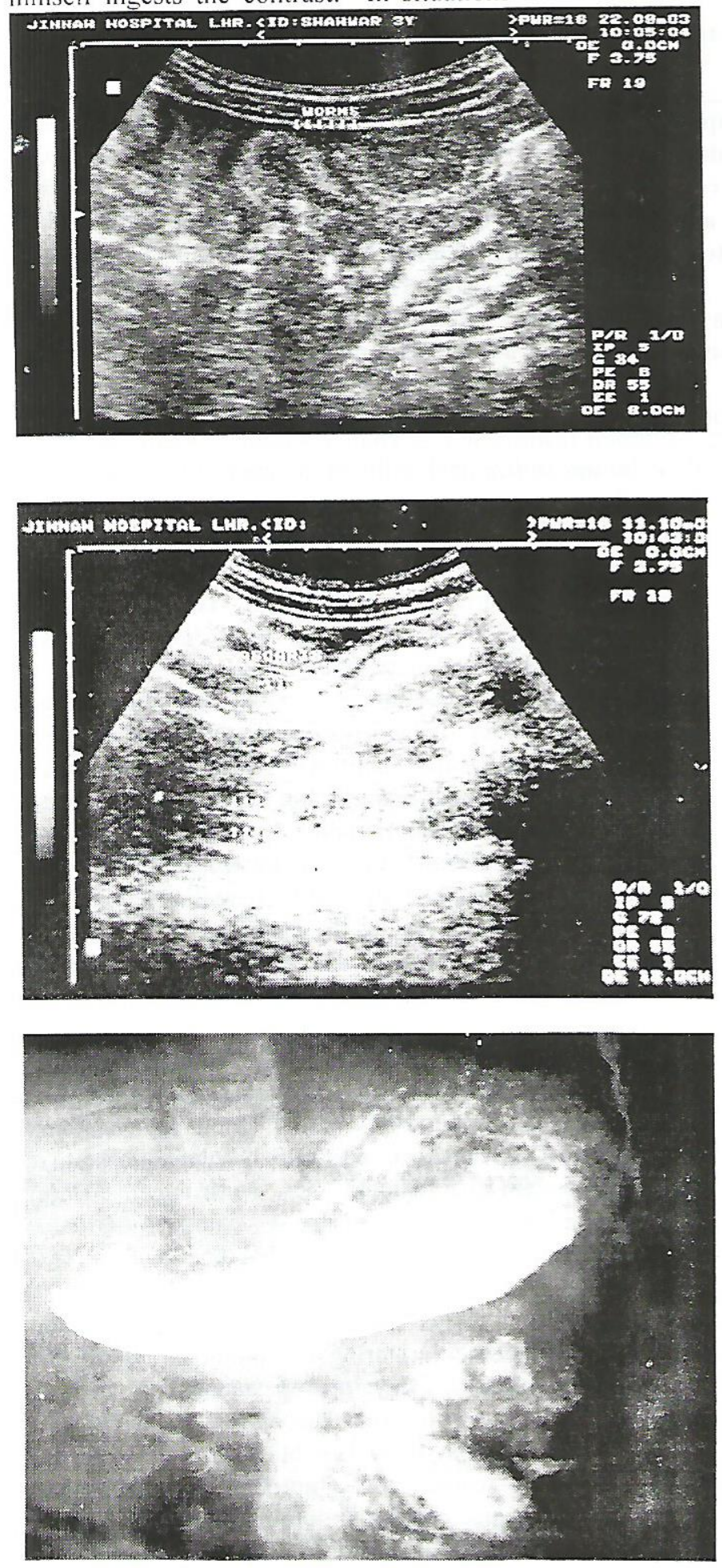

study is contraindicated USG can be a good tool to demonstrate pathology. Majority of patients can be managed conservatively because these worms disintegrate and shrivel up. Patients get relief from symptoms.

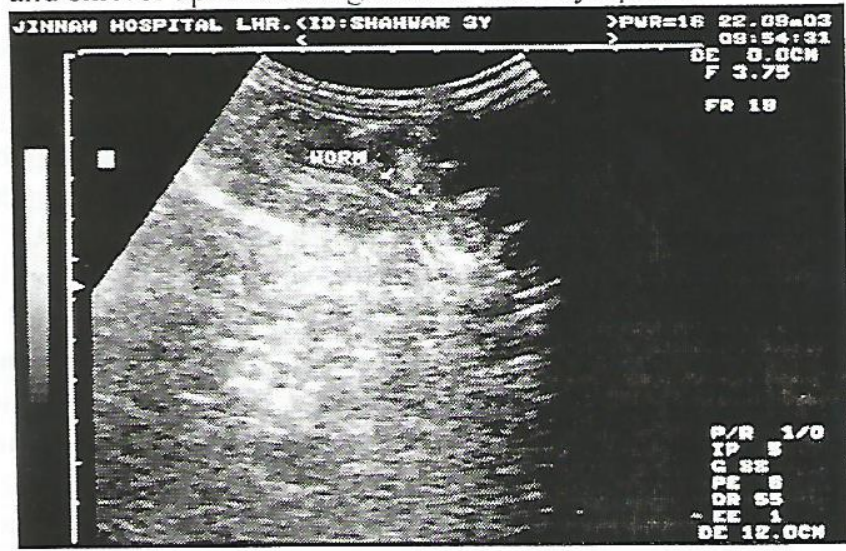

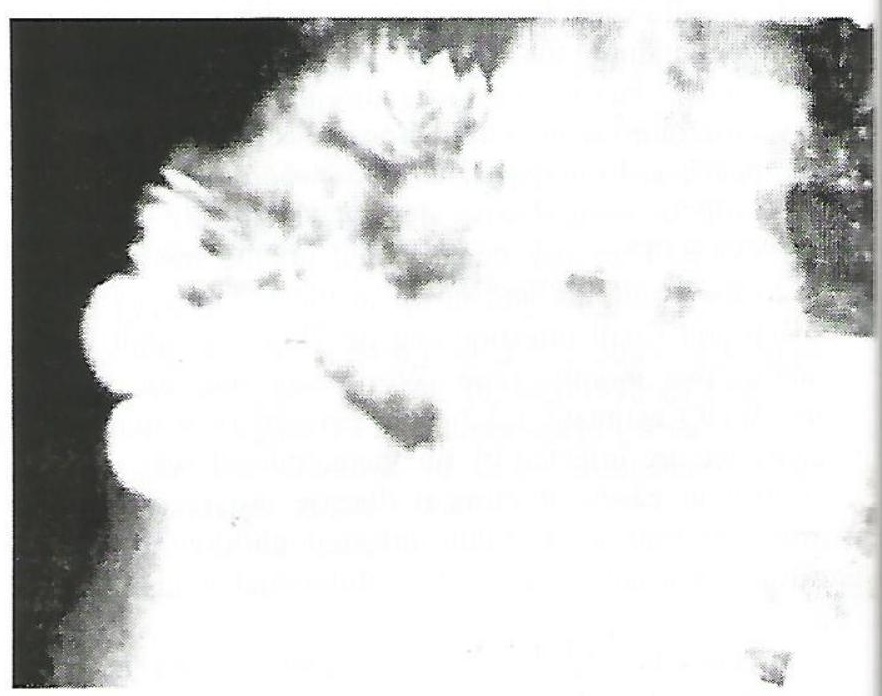




\section{Conclusion}

Worm infestation has never been taken seriously as cause of abdominal pain due to high prevalence even in asymptomatic patients. We hereby conclude that USG permits early diagnosis and prompt treatment, hence stressing upon the efficacy and importance of USG in diagnosis of intestinal ascariasis.

Improvement in sanitation plays a vital role in the control of this parasitic disease. Sonographic examination of abdomen in patients with ascariasis is predominant especially in paediatric age group. Even after finding the normal major viscera of abdomen, one should magnify the system and search for this parasite in bowel and unwanted surgical exploration can be avoided.

\section{References}

1. Peck RJ. Ultrasonography of intestinal ascaris. Journal of Clinical Ultrasound. 1990: 133: 741-43.

2. Scheible W. Goldberger LE. Diagnosis of small bowel obstruction: The contribution of diagnostic ultrasound. American Journal of Radiology 1979: 133: 685-88.

3. Ishtyak Ahmad Mir. Sonographic Appearance In Intestinal Ascariasis JK-Practitioner 2002; 9(4): 234-235.

4. Fauci AS et al.. Harrison's Principles of Internal Medicine. McGraw Hill. 1998:1208-1210.

5. Blacklock and Southwell A Guide to Human Parasitology.pg 120 to 124 .

6. Medical Microbiology and Immunology By Warren Levinson and Ernest Jawetz 343 - 348 . 